\title{
Note on orbifold Chow ring of semi-projective toric Deligne-Mumford stacks
}

\author{
Yunfeng Jiang AND Hsian-Hua Tseng
}

\begin{abstract}
We prove a formula for the orbifold Chow ring of semi-projective toric Deligne-Mumford (DM) stacks, generalizing the orbifold Chow ring formula of projective toric DM stacks by Borisov-ChenSmith. We also consider a special kind of semi-projective toric DM stacks, the Lawrence toric DM stacks. We prove that the orbifold Chow ring of a Lawrence toric DM stack is isomorphic to the orbifold Chow ring of its associated hypertoric DM stack studied in Y. Jiang and H.-H. Tseng, The orbifold Chow ring of hypertoric Deligne-Mumford stacks, J. Reine Angew. Math. (Crelle's Journal), to appear, math.AG/0512199.
\end{abstract}

\section{Introduction}

The main aim of this paper is to generalize the orbifold Chow ring formula of Borisov-Chen-Smith for projective toric Deligne-Mumford (DM) stacks to the case of semi-projective toric DM stacks.

In the paper [3], Borisov, Chen and Smith developed the theory of toric DM stacks using stacky fans, generalizing the quotient construction of simplicial toric varieties, $[7,8]$. A stacky fan is a triple $\boldsymbol{\Sigma}=(N, \Sigma, \beta)$, where $N$ is a finitely generated abelian group, $\Sigma$ is a simplicial fan in the lattice $\bar{N}:=N /$ torsion and $\beta: \mathbb{Z}^{n} \rightarrow N$ is a map given by a collection of vectors $\left\{b_{1}, \ldots, b_{n}\right\} \subset N$ such that the images $\left\{\bar{b}_{1}, \ldots, \bar{b}_{n}\right\}$ generate the fan $\Sigma$. A toric DM stack $\mathcal{X}(\boldsymbol{\Sigma})$ is defined using $\boldsymbol{\Sigma}$; it is a quotient stack whose coarse moduli space is the toric variety $X(\Sigma)$ corresponding to the simplicial fan $\Sigma$.

The construction of toric DM stacks was slightly generalized later in [12], in which the notion of extended stacky fans was introduced. This new notion is based on that of stacky fans plus some extra data. Extended stacky fans yield toric DM stacks in the same way as stacky fans do. The main point is that extended stacky fans provide presentations of toric DM stacks not available from stacky fans.

When $X(\Sigma)$ is projective, it is found in [3] that the orbifold Chow ring (or Chen-Ruan cohomology ring) of $\mathcal{X}(\boldsymbol{\Sigma})$ introduced in $[1,5]$ is isomorphic 
to a deformed ring of the group ring of $N$. We call a toric DM stack $\mathcal{X}(\boldsymbol{\Sigma})$ semi-projective if its coarse moduli space $X(\Sigma)$ is semi-projective. Hausel and Sturmfels [10] computed the Chow ring of semi-projective toric varieties. Their answer is also known as the "Stanley-Reisner" ring of a fan, also see $[14,15]$. Using their result, we prove a formula of the orbifold Chow ring of semi-projective toric DM stacks.

Consider an extended stacky fan $\boldsymbol{\Sigma}=(N, \Sigma, \beta)$, where $\Sigma$ is the simplicial fan of the semi-projective toric variety $X(\Sigma)$. Let $N_{\text {tor }}$ be the torsion subgroup of $N$, then $N=\bar{N} \oplus N_{\text {tor }}$. Let $N_{\Sigma}:=|\Sigma| \oplus N_{\text {tor. }}$. Note that $|\Sigma|$ is convex, and so $|\Sigma| \oplus N_{\text {tor }}$ is a subgroup of $N$. Define the deformed ring $\mathbb{Q}\left[N_{\Sigma}\right]:=\bigoplus_{c \in N_{\Sigma}} \mathbb{Q} y^{c}$ with the product structure given by

$$
y^{c_{1}} \cdot y^{c_{2}}:= \begin{cases}y^{c_{1}+c_{2}} & \text { if there is a cone } \sigma \in \Sigma \text { such that } \bar{c}_{1} \in \sigma, \bar{c}_{2} \in \sigma ; \\ 0 & \text { otherwise. }\end{cases}
$$

Note that if $\mathcal{X}(\boldsymbol{\Sigma})$ is projective, then $N_{\Sigma}=N$ and $\mathbb{Q}\left[N_{\Sigma}\right]$ is the deformed ring $\mathbb{Q}[N]^{\Sigma}$ in [3]. Let $A_{\text {orb }}^{*}(\mathcal{X}(\boldsymbol{\Sigma}))$ denote the orbifold Chow ring of the toric DM stack $\mathcal{X}(\boldsymbol{\Sigma})$.

Theorem 1.1. Assume that $\mathcal{X}(\boldsymbol{\Sigma})$ is semi-projective. There is an isomorphism of rings

$$
A_{\mathrm{orb}}^{*}(\mathcal{X}(\boldsymbol{\Sigma})) \cong \frac{\mathbb{Q}\left[N_{\Sigma}\right]}{\left\{\sum_{i=1}^{n} e\left(b_{i}\right) y^{b_{i}}: e \in N^{\star}\right\}} .
$$

The strategy of proving Theorem 1.1 is as follows. We use a formula in [10] for the ordinary Chow ring of semi-projective toric varieties. We prove that each twisted sector is also a semi-projective toric DM stack. With this, we use a method similar to that in $[3,12]$ to prove the isomorphism as modules. The argument to show the isomorphism as rings is the same as that in [3], except that we only take elements in the support of the fan.

An interesting class of examples of semi-projective toric DM stack is the Lawrence toric DM stacks. We discuss the properties of such stacks. We study the 3-twisted sectors or twisted sectors of Lawrence toric DM stacks, which allows us to draw connections to hypertoric DM stacks studied in [13]. We prove that the orbifold Chow ring of a Lawrence toric DM stack is isomorphic to the orbifold Chow ring of its associated hypertoric DM stack. This is an analog of Theorem 1.1 in [10] for orbifold Chow rings. 
The rest of this text is organized as follows. In Section 2, we define semiprojective toric DM stacks and prove Theorem 1.1. Results on Lawrence toric DM stacks are discussed in Section 3.

Conventions. In this paper we work entirely algebraically over the field of complex numbers. Chow rings and orbifold Chow rings are taken with rational coefficients. By an orbifold we mean a smooth DM stack with trivial generic stabilizer.

For a simplicial fan $\Sigma$, we use $|\Sigma|$ to represent the lattice points in $\Sigma$. Note that if $\Sigma$ is convex, $|\Sigma|$ is a free abelian subgroup of $N$. We write $N^{\star}$ for $\operatorname{Hom}_{\mathbb{Z}}(N, \mathbb{Z})$ and $N \rightarrow \bar{N}$ the natural map of modding out torsions. We refer to [3] for the construction of the Gale dual $\beta^{\vee}: \mathbb{Z}^{m} \rightarrow \operatorname{DG}(\beta)$ of $\beta: \mathbb{Z}^{m} \rightarrow N$.

\section{Semi-projective toric DM stacks and their orbifold chow rings}

In this section, we define semi-projective toric DM stacks and discuss their properties.

\subsection{Semi-projective toric DM stacks}

Definition 2.1 [10]. A toric variety $X$ is called semi-projevtive if the natural map

$$
\pi: X \rightarrow X_{0}=\operatorname{Spec}\left(H^{0}\left(X, \mathcal{O}_{X}\right)\right)
$$

is projective and $X$ has at least one torus-fixed point.

Definition 2.2 [12]. An extended stacky fan $\boldsymbol{\Sigma}$ is a triple $(N, \Sigma, \beta)$, where $N$ is a finitely generated abelian group, $\Sigma$ is a simplicial fan in $N_{\mathbb{R}}$ and $\beta: \mathbb{Z}^{m} \rightarrow N$ is the map determined by the elements $\left\{b_{1}, \ldots, b_{m}\right\}$ in $N$ such that $\left\{\bar{b}_{1}, \ldots, \bar{b}_{n}\right\}$ generate the simplicial fan $\Sigma$ (here $m \geq n$ ).

Given an extended stacky fan $\boldsymbol{\Sigma}=(N, \Sigma, \beta)$, we have the following exact sequences:

$$
\begin{gathered}
0 \longrightarrow \operatorname{DG}(\beta)^{\star} \longrightarrow \mathbb{Z}^{m} \stackrel{\beta}{\longrightarrow} N \longrightarrow \operatorname{Coker}(\beta) \longrightarrow 0 \\
0 \longrightarrow N^{\star} \longrightarrow \mathbb{Z}^{m} \stackrel{\beta^{\vee}}{\longrightarrow} \operatorname{DG}(\beta) \longrightarrow \operatorname{Coker}\left(\beta^{\vee}\right) \longrightarrow 0
\end{gathered}
$$


where $\beta^{\vee}$ is the Gale dual of $\beta$ (see [3]). Applying $\operatorname{Hom}_{\mathbb{Z}}\left(-, \mathbb{C}^{*}\right)$ to $(2.2)$ yields

$$
1 \longrightarrow \mu \longrightarrow G \stackrel{\alpha}{\longrightarrow}\left(\mathbb{C}^{*}\right)^{m} \longrightarrow\left(\mathbb{C}^{*}\right)^{d} \longrightarrow 1
$$

The toric DM stack $\mathcal{X}(\boldsymbol{\Sigma})$ is the quotient stack $[Z / G]$, where $Z:=\left(\mathbb{C}^{n} \backslash\right.$ $\left.V\left(J_{\Sigma}\right)\right) \times\left(\mathbb{C}^{*}\right)^{m-n}, J_{\Sigma}$ is the irrelevant ideal of the fan $\Sigma$ and $G$ acts on $Z$ through the map $\alpha$ in (2.3). The coarse moduli space of $\mathcal{X}(\boldsymbol{\Sigma})$ is the simplicial toric variety $X(\Sigma)$ corresponding to the simplicial fan $\Sigma$; see $[3,12]$.

Definition 2.3. A toric DM stack $\mathcal{X}(\boldsymbol{\Sigma})$ is semi-projective if the coarse moduli space $X(\Sigma)$ is semi-projective.

Theorem 2.4. The following notions are equivalent:

(1) A semi-projective toric DM stack $\mathcal{X}(\boldsymbol{\Sigma})$;

(2) A toric DM stack $\mathcal{X}(\boldsymbol{\Sigma})$ such that the simplicial fan $\Sigma$ is a regular triangulation of $\mathcal{B}=\left\{\bar{b}_{1}, \ldots, \bar{b}_{n}\right\}$ which spans the lattice $\bar{N}$.

Proof. Since the toric DM stack is semi-projective if its coarse moduli space is semi-projective, the theorem follows from results in [10].

\subsection{The inertia stack}

Let $\boldsymbol{\Sigma}$ be an extended stacky fan and $\sigma \in \Sigma$ a cone. Define $\operatorname{link}(\sigma):=$ $\{\tau: \sigma+\tau \in \Sigma, \sigma \cap \tau=0\}$. Let $\left\{\widetilde{\rho}_{1}, \ldots, \widetilde{\rho}_{l}\right\}$ be the rays in $\operatorname{link}(\sigma)$. Consider the quotient extended stacky fan $\Sigma / \sigma=(N(\sigma), \Sigma / \sigma, \beta(\sigma))$, with $\beta(\sigma)$ : $\mathbb{Z}^{l+m-n} \rightarrow N(\sigma)$ given by the images of $b_{1}, \ldots, b_{l}$ and $b_{n+1}, \ldots, b_{m}$ under $N \rightarrow N(\sigma)$. By the construction of toric DM stacks, if $\sigma$ is contained in a top-dimensional cone in $\Sigma$, we have $\mathcal{X}(\boldsymbol{\Sigma} / \sigma):=[Z(\sigma) / G(\sigma)]$, where $Z(\sigma)=\left(\mathbb{A}^{l} \backslash \mathbb{V}\left(J_{\Sigma / \sigma}\right)\right) \times\left(\mathbb{C}^{*}\right)^{m-n}$ and $G(\sigma)=\operatorname{Hom}_{\mathbb{Z}}\left(\operatorname{DG}(\beta(\sigma)), \mathbb{C}^{*}\right)$.

Lemma 2.5. If $\mathcal{X}(\boldsymbol{\Sigma})$ is semi-projective, so is $\mathcal{X}(\boldsymbol{\Sigma} / \sigma)$.

Proof. Semi-projectivity of the stack $\mathcal{X}(\boldsymbol{\Sigma})$ means the simplicial fan $\Sigma$ is a fan coming from a regular triangulation of $\mathcal{B}=\left\{\bar{b}_{1}, \ldots, \bar{b}_{n}\right\}$ which spans the lattice $\bar{N}$. Let $\operatorname{pos}(\mathcal{B})$ be the convex polyhedral cone generated by $\mathcal{B}$. Then from [10], the triangulation is supported on $\operatorname{pos}(\mathcal{B})$ and is determined by a simple polyhedron whose normal fan is $\Sigma$. So $\sigma$ is contained in a top-dimensional cone $\tau$ in $\Sigma$. The image $\widetilde{\tau}$ of $\tau$ under quotient by $\sigma$ is a top-dimensional cone in the quotient fan $\Sigma / \sigma$. So the toric variety $X(\Sigma / \sigma)$ 
is semi-projective by Theorem 2.4, and the stack $\mathcal{X}(\boldsymbol{\Sigma} / \sigma)$ is semi-projective by definition.

Recall in [3] that for each top-dimensional cone $\sigma$ in $\Sigma$, define $\operatorname{Box}(\sigma)$ to be the set of elements $v \in N$ such that $\bar{v}=\sum_{\rho_{i} \subseteq \sigma} a_{i} \bar{b}_{i}$ for some $0 \leq a_{i}<1$. Elements in $\operatorname{Box}(\sigma)$ are in one-to-one correspondence with elements in the finite group $N(\sigma)=N / N_{\sigma}$, where $N(\sigma)$ is a local group of the stack $\mathcal{X}(\boldsymbol{\Sigma})$. In fact, we write $\bar{v}=\sum_{\rho_{i} \subseteq \sigma(\bar{v})} a_{i} \bar{b}_{i}$ for some $0<a_{i}<1$, where $\sigma(\bar{v})$ is the minimal cone containing $\bar{v}$. We denote by $\operatorname{Box}(\boldsymbol{\Sigma})$ the union of $\operatorname{Box}(\sigma)$ for all top-dimensional cones $\sigma$.

Proposition 2.6. The r-inertia stack is given by

$$
\mathcal{I}_{r}(\mathcal{X}(\boldsymbol{\Sigma}))=\coprod_{\left(v_{1}, \ldots, v_{r}\right) \in B o x(\boldsymbol{\Sigma})^{r}} \mathcal{X}\left(\boldsymbol{\Sigma} / \sigma\left(\bar{v}_{1}, \ldots, \bar{v}_{r}\right)\right),
$$

where $\sigma\left(\bar{v}_{1}, \ldots, \bar{v}_{r}\right)$ is the minimal cone in $\Sigma$ containing $\bar{v}_{1}, \ldots, \bar{v}_{r}$.

Proof. Since $G$ is an abelian group, we have

$$
\mathcal{I}_{r}(\mathcal{X}(\boldsymbol{\Sigma}))=\left[\left(\coprod_{\left(v_{1}, \ldots, v_{r}\right) \in(G)^{r}} Z^{\left(v_{1}, \ldots, v_{r}\right)}\right) / G\right]
$$

where $Z^{\left(v_{1}, \ldots, v_{r}\right)} \subset Z$ is the subvariety fixed by $v_{1}, \ldots, v_{r}$. Since $\sigma\left(\bar{v}_{1}, \ldots, \bar{v}_{r}\right)$ is contained in a top-dimensional cone in $\Sigma$. We use the same method as in Lemma 4.6 and Proposition 4.7 of [3] to prove that $\left[Z^{\left(v_{1}, \ldots, v_{r}\right)} / G\right] \cong$ $\mathcal{X}\left(\Sigma / \sigma\left(\bar{v}_{1}, \ldots, \bar{v}_{r}\right)\right)$.

Note that in (2.4) each component is semi-projective.

\subsection{The orbifold Chow ring}

In this section, we compute the orbifold Chow ring of semi-projective toric DM stacks and prove Theorem 1.1.

The module structure. Let $\boldsymbol{\Sigma}=(N, \Sigma, \beta)$ be an extended stacky fan such that the toric DM stack $\mathcal{X}(\boldsymbol{\Sigma})$ is semi-projective. Since the fan $\Sigma$ is convex, $|\Sigma|$ is an abelian subgroup of $N$. We put $N_{\Sigma}:=|\Sigma| \oplus N_{\text {tor }}$, where $N_{\text {tor }}$ is the torsion subgroup of $N$. Define the deformed ring $\mathbb{Q}\left[N_{\Sigma}\right]:=\bigoplus_{c \in N_{\Sigma}} \mathbb{Q} y^{c}$ with the product structure given by (1.1).

Let $\left\{\rho_{1}, \ldots, \rho_{n}\right\}$ be the rays of $\Sigma$, then each $\rho_{i}$ corresponds to a line bundle $L_{i}$ over the toric DM stack $\mathcal{X}(\boldsymbol{\Sigma})$ given by the trivial line bundle 
$\mathbb{C} \times Z$ over $Z$ with the $G$ action on $\mathbb{C}$ given by the $i$ th component $\alpha_{i}$ of $\alpha: G \rightarrow\left(\mathbb{C}^{*}\right)^{m}$ in (2.3). The first Chern classes of the line bundles $L_{i}$, which we identify with $y^{b_{i}}$, generate the cohomology ring of the simplicial toric variety $X(\Sigma)$.

Let $S_{\boldsymbol{\Sigma}}$ be the quotient ring $\mathbb{Q}\left[y^{b_{1}}, \ldots, y^{b_{n}}\right] / I_{\Sigma}$, where $I_{\Sigma}$ is the squarefree ideal of the fan $\Sigma$ generated by the monomials

$$
\left\{y^{b_{i_{1}}} \cdots y^{b_{i_{k}}}: \bar{b}_{i_{1}}, \ldots, \bar{b}_{i_{k}} \text { do not generate a cone in } \Sigma\right\} \text {. }
$$

It is clear that $S_{\Sigma}$ is a subring of the deformed ring $\mathbb{Q}\left[N_{\Sigma}\right]$.

Lemma 2.7. Let $A^{*}(\mathcal{X}(\boldsymbol{\Sigma}))$ be the ordinary Chow ring of a semi-projective toric DM stack $\mathcal{X}(\boldsymbol{\Sigma})$. Then there is a ring isomorphism:

$$
A^{*}(\mathcal{X}(\boldsymbol{\Sigma})) \cong \frac{S_{\boldsymbol{\Sigma}}}{\left\{\sum_{i=1}^{n} e\left(b_{i}\right) y^{b_{i}}: e \in N^{\star}\right\}} .
$$

Proof. The lemma is easily proven from the fact that the Chow ring of a DM stack is isomorphic to the Chow ring of its coarse moduli space [17]; [10, Proposition 2.11].

Now we study the module structure on $A_{\text {orb }}^{*}(\mathcal{X}(\Sigma))$. Because $\Sigma$ is a simplicial fan, we have:

Lemma 2.8. For any $c \in N_{\Sigma}$, let $\sigma$ be the minimal cone in $\Sigma$ containing $\bar{c}$. Then there is a unique expression $c=v+\sum_{\rho_{i} \subset \sigma} m_{i} b_{i}$, where $m_{i} \in \mathbb{Z}_{\geq 0}$ and $v \in \operatorname{Box}(\sigma)$.

Proposition 2.9. Let $\mathcal{X}(\boldsymbol{\Sigma})$ be a semi-projective toric DM stack associated to an extended stacky fan $\boldsymbol{\Sigma}$. We have an isomorphism of $A^{*}(\mathcal{X}(\boldsymbol{\Sigma}))$ modules:

$$
\bigoplus_{v \in \operatorname{Box}(\boldsymbol{\Sigma})} A^{*}(\mathcal{X}(\boldsymbol{\Sigma} / \sigma(\bar{v})))\left[\operatorname{deg}\left(y^{v}\right)\right] \cong \frac{\mathbb{Q}\left[N_{\Sigma}\right]}{\left\{\sum_{i=1}^{n} e\left(b_{i}\right) y^{b_{i}}: e \in N^{\star}\right\}}
$$

Proof. From the definition of $\mathbb{Q}\left[N_{\Sigma}\right]$ and Lemma 2.8, we see that $\mathbb{Q}\left[N_{\Sigma}\right]=$ $\bigoplus_{v \in \operatorname{Box}(\boldsymbol{\Sigma})} y^{v} \cdot S_{\boldsymbol{\Sigma}}$. The rest is similar to the proof of [12, Proposition 4.7], we leave it to the readers.

The Chen-Ruan product structure. The orbifold cup product on a DM stack $\mathcal{X}$ is defined using genus 0, degree 0, 3-pointed orbifold GromovWitten invariants on $\mathcal{X}$. The relevant moduli space is the disjoint union of 
all 3-twisted sectors (i.e., the double inertia stack). By (2.4), the 3-twisted sectors of a semi-projective toric DM stack $\mathcal{X}(\boldsymbol{\Sigma})$ are

$$
\coprod_{\left(v_{1}, v_{2}, v_{3}\right) \in \operatorname{Box}(\boldsymbol{\Sigma})^{3}, v_{1} v_{2} v_{3}=1} \mathcal{X}\left(\boldsymbol{\Sigma} / \sigma\left(\bar{v}_{1}, \bar{v}_{2}, \bar{v}_{3}\right)\right) .
$$

Let $\mathrm{ev}_{i}: \mathcal{X}\left(\boldsymbol{\Sigma} / \sigma\left(\bar{v}_{1}, \bar{v}_{2}, \bar{v}_{3}\right)\right) \rightarrow \mathcal{X}\left(\boldsymbol{\Sigma} / \sigma\left(\bar{v}_{i}\right)\right)$ be the evaluation maps. The obstruction bundle (see $[5,6]) \mathrm{Ob}_{\left(v_{1}, v_{2}, v_{3}\right)}$ over the 3-twisted sector $\mathcal{X}\left(\boldsymbol{\Sigma} / \sigma\left(\bar{v}_{1}, \bar{v}_{2}, \bar{v}_{3}\right)\right)$ are defined by

$$
\mathrm{Ob}_{\left(v_{1}, v_{2}, v_{3}\right)}:=\left(e^{*} T(\mathcal{X}(\boldsymbol{\Sigma})) \otimes H^{1}\left(C, \mathcal{O}_{C}\right)\right)^{H},
$$

where $e: \mathcal{X}\left(\boldsymbol{\Sigma} / \sigma\left(\bar{v}_{1}, \bar{v}_{2}, \bar{v}_{3}\right)\right) \rightarrow \mathcal{X}(\boldsymbol{\Sigma})$ is the embedding, $C \rightarrow \mathbb{P}^{1}$ is the $H$-covering branched over three marked points $\{0,1, \infty\} \subset \mathbb{P}^{1}$, and $H$ is the group generated by $v_{1}, v_{2}, v_{3}$.

A general result in $[4,11]$ about the obstruction bundle implies the following.

Proposition 2.10. Let $\mathcal{X}\left(\boldsymbol{\Sigma} / \sigma\left(\bar{v}_{1}, \bar{v}_{2}, \bar{v}_{3}\right)\right)$ be a 3 -twisted sector of the stack $\mathcal{X}(\boldsymbol{\Sigma})$. Suppose $v_{1}+v_{2}+v_{3}=\sum_{\rho_{i} \subset \sigma\left(\bar{v}_{1}, \bar{v}_{2}, \bar{v}_{3}\right)} a_{i} b_{i}, a_{i}=1$ or 2 . Then the Euler class of the obstruction bundle $\mathrm{Ob}_{\left(v_{1}, v_{2}, v_{3}\right)}$ on $\mathcal{X}\left(\boldsymbol{\Sigma} / \sigma\left(\bar{v}_{1}, \bar{v}_{2}, \bar{v}_{3}\right)\right)$ is

$$
\left.\prod_{a_{i}=2} c_{1}\left(L_{i}\right)\right|_{\mathcal{X}\left(\boldsymbol{\Sigma} / \sigma\left(\bar{v}_{1}, \bar{v}_{2}, \bar{v}_{3}\right)\right)}
$$

where $L_{i}$ is the line bundle over $\mathcal{X}(\boldsymbol{\Sigma})$ corresponding to the ray $\rho_{i}$.

Let $v \in \operatorname{Box}(\boldsymbol{\Sigma})$, say $v \in N(\sigma)$, for some top-dimensional cone $\sigma$. Let $\check{v} \in$ $\operatorname{Box}(\boldsymbol{\Sigma})$ be the inverse of $v$ as an element in the group $N(\sigma)$. Equivalently, if $v=\sum_{\rho_{i} \subseteq \sigma(\bar{v})} \alpha_{i} b_{i}$ for $0<\alpha_{i}<1$, then $\check{v}=\sum_{\rho_{i} \subseteq \sigma(\bar{v})}\left(1-\alpha_{i}\right) b_{i}$. Then for $\alpha_{1}, \alpha_{2} \in A_{\text {orb }}^{*}(\mathcal{X}(\boldsymbol{\Sigma}))$, the orbifold cup product is defined by

$$
\alpha_{1} \cup_{\mathrm{orb}} \alpha_{2}=\widehat{\mathrm{ev}}_{3 *}\left(\operatorname{ev}_{1}^{*} \alpha_{1} \cup \mathrm{ev}_{2}^{*} \alpha_{2} \cup e\left(\mathrm{Ob}_{\left(v_{1}, v_{2}, v_{3}\right)}\right)\right)
$$

where $\widehat{\mathrm{ev}}_{3}=I \circ \mathrm{ev}_{3}$, and $I: \mathcal{I} \mathcal{X}(\boldsymbol{\Sigma}) \rightarrow \mathcal{I} \mathcal{X}(\boldsymbol{\Sigma})$ is the natural map given by $(x, g) \mapsto\left(x, g^{-1}\right)$.

Proof of Theorem 1.1. By Proposition 2.9, it remains to consider the cup product. In this case, for any $v_{1}, v_{2} \in \operatorname{Box}(\boldsymbol{\Sigma})$, we also have

$$
v_{1}+v_{2}=\check{v}_{3}+\sum_{a_{i}=2} b_{i}+\sum_{i \in J} b_{i},
$$


where $J$ represents the set of $j$ such that $\rho_{j}$ belongs to $\sigma\left(\bar{v}_{1}, \bar{v}_{2}\right)$, but not belong to $\sigma\left(\bar{v}_{3}\right)$. Then the proof is the same as the proof in [3]. We omit the details.

\section{Lawrence toric DM stacks}

In this section, we study a special type of semi-projective toric DM stacks called the Lawrence toric DM stacks. Their orbifold Chow rings are shown to be isomorphic to the orbifold Chow rings of their associated hypertoric DM stacks studied in [13].

\subsection{Stacky hyperplane arrangements}

Let $N,\left\{b_{1}, \ldots, b_{m}\right\} \in N, \beta: \mathbb{Z}^{m} \rightarrow N$, and $\left\{\bar{b}_{1}, \ldots, \bar{b}_{m}\right\} \subset \bar{N}$ be as in Definition 2.2. We assume that $\left\{b_{1}, \ldots, b_{m}\right\} \in N$ are nontorsion integral vectors. We still have the exact sequences (2.1) and (2.2). The Gale dual map $\beta^{\vee}$ of $\beta$ is given by a collection of integral vectors $\beta^{\vee}=\left(a_{1}, \ldots, a_{m}\right)$. Choose a generic element $\theta \in \mathrm{DG}(\beta)$ and let $\psi:=\left(r_{1}, \ldots, r_{m}\right)$ be a lifting of $\theta$ in $\mathbb{Z}^{m}$ such that $\theta=-\beta^{\vee} \psi$. Note that $\theta$ is generic if and only if it is not in any hyperplane of the configuration determined by $\beta^{\vee}$ in $\operatorname{DG}(\beta)_{\mathbb{R}}$. Associated to $\theta$ there is a hyperplane arrangement $\mathcal{H}=\left\{H_{1}, \ldots, H_{m}\right\}$ defined as follows: let $H_{i}$ be the hyperplane

$$
H_{i}:=\left\{v \in M_{\mathbb{R}} \mid<\bar{b}_{i}, v>+r_{i}=0\right\} \subset M_{\mathbb{R}}
$$

This determines hyperplane arrangement in $M_{\mathbb{R}}$, up to translation. It is well known that hyperplane arrangements determine the topology of hypertoric varieties [2]. We call $\mathcal{A}:=(N, \beta, \theta)$ a stacky hyperplane arrangement.

The toric variety $X(\Sigma)$ is defined by the weighted polytope $\boldsymbol{\Gamma}:=\bigcap_{i=1}^{m} F_{i}$, where $F_{i}=\left\{v \in M_{\mathbb{R}} \mid\left\langle b_{i}, v\right\rangle+r_{i} \geq 0\right\}$. Suppose that $\boldsymbol{\Gamma}$ is bounded, the fan $\Sigma$ is the normal fan of $\Gamma$ in $M_{\mathbb{R}}=\mathbb{R}^{d}$ with one dimensional rays generated by $\bar{b}_{1}, \ldots, \bar{b}_{n}$. By reordering, we may assume that $H_{1}, \ldots, H_{n}$ are the hyperplanes that bound the polytope $\boldsymbol{\Gamma}$, and $H_{n+1}, \ldots, H_{m}$ are the other hyperplanes. Then we have an extended stacky fan $\boldsymbol{\Sigma}=(N, \Sigma, \beta)$ as in Definition 2.2, with $\Sigma$ the normal fan of $\boldsymbol{\Gamma}, \beta$ : $\mathbb{Z}^{m} \rightarrow N$ given by $\left\{b_{1}, \ldots, b_{n}, b_{n+1}, \ldots, b_{m}\right\} \subset N$, and $\left\{b_{n+1}, \ldots, b_{m}\right\}$ the extra data. We define the hypertoric DM stack $\mathcal{M}(\mathcal{A})$ using this $\mathcal{A}$; see [13] for more details. 


\subsection{Lawrence toric DM stacks}

Applying Gale dual to the map

$$
\mathbb{Z}^{m} \oplus \mathbb{Z}^{m} \rightarrow \operatorname{DG}(\beta)
$$

given by $\left(\beta^{\vee},-\beta^{\vee}\right)$, we obtain

$$
\beta_{\mathrm{L}}: \mathbb{Z}^{m} \oplus \mathbb{Z}^{m} \longrightarrow N_{\mathrm{L}}
$$

which is given by integral vectors $\left\{b_{\mathrm{L}, 1}, \ldots, b_{\mathrm{L}, m}, b_{\mathrm{L}, 1}^{\prime}, \ldots, b_{\mathrm{L}, m}^{\prime}\right\}$ in $N_{\mathrm{L}}$. The natural images $\left\{\bar{b}_{\mathrm{L}, 1}, \ldots, \bar{b}_{\mathrm{L}, m}, \bar{b}_{\mathrm{L}, 1}^{\prime}, \ldots, \bar{b}_{\mathrm{L}, m}^{\prime}\right\} \subset \bar{N}_{\mathrm{L}}$ are called the Lawrence lifting of $\left\{\bar{b}_{1}, \ldots, \bar{b}_{m}\right\} \subset \bar{N}$.

Associated to the generic element $\theta$, let $\bar{\theta}$ be the natural image under the map $\operatorname{DG}(\beta) \rightarrow \overline{\mathrm{DG}(\beta)}$. Then the map $\bar{\beta}^{\vee}: \mathbb{Z}^{m} \rightarrow \overline{\mathrm{DG}(\beta)}$ is given by $\bar{\beta}^{\vee}=\left(\bar{a}_{1}, \ldots, \bar{a}_{m}\right)$. For any column basis of the form $C=\left\{\bar{a}_{i_{1}}, \ldots, \bar{a}_{i_{m-d}}\right\}$, there exist unique $\lambda_{1}, \ldots, \lambda_{m-d}$ such that

$$
a_{i_{1}} \lambda_{1}+\cdots+a_{i_{m-d}} \lambda_{m-d}=\bar{\theta}
$$

Let $\mathbb{C}\left[z_{1}, \ldots, z_{m}, w_{1}, \ldots, w_{m}\right]$ be the coordinate ring of $\mathbb{C}^{2 m}$. Let $\sigma(C, \theta)=$ $\left\{\bar{b}_{i_{j}} \mid \lambda_{j}>0\right\} \sqcup\left\{\bar{b}_{i_{j}}^{\prime} \mid \lambda_{j}<0\right\}$, and $C(\theta)=\left\{z_{i_{j}} \mid \lambda_{j}>0\right\} \sqcup\left\{w_{i_{j}} \mid \lambda_{j}<0\right\}$. We set

$$
\left.\mathcal{I}_{\theta}:=\left\langle\prod C(\theta)\right| C \text { is a column basis of } \bar{\beta}^{\vee}\right\rangle
$$

and

$$
\Sigma_{\theta}:=\left\{\bar{\sigma}(C, \theta): C \text { is a column basis of } \bar{\beta}^{\vee}\right\},
$$

where $\bar{\sigma}(C, \theta)=\left\{\bar{b}_{\mathrm{L}, 1}, \ldots, \bar{b}_{\mathrm{L}, m}, \bar{b}_{\mathrm{L}, 1}^{\prime}, \cdots, \bar{b}_{\mathrm{L}, m}^{\prime}\right\} \backslash \sigma(C, \theta)$ is the complement of $\sigma(C, \theta)$ and corresponds to the maximal cones in $\Sigma_{\theta}$. According to [10], $\Sigma_{\theta}$ is the fan of Lawrence toric variety $X\left(\Sigma_{\theta}\right)$ corresponding to $\theta$ in the lattice $\bar{N}_{L}$. The ideal $\mathcal{I}_{\theta}$ is the irrelevant ideal of the fan $\Sigma_{\theta}$. Then we have the Lawrence stacky fan $\boldsymbol{\Sigma}_{\boldsymbol{\theta}}=\left(N_{L}, \Sigma_{\theta}, \beta_{L}\right)$ introduced in [13].

Applying $\operatorname{Hom}_{\mathbb{Z}}\left(-, \mathbb{C}^{*}\right)$ functor to $(3.2)$, we get

$$
\alpha_{h}: G \rightarrow\left(\mathbb{C}^{*}\right)^{2 m}
$$

So $G$ acts on $\mathbb{C}^{2 m}$ through $\alpha_{h}$. From Section $2, \mathcal{X}\left(\boldsymbol{\Sigma}_{\boldsymbol{\theta}}\right)=\left[\left(\mathbb{C}^{2 m} \backslash\right.\right.$ $\left.V\left(\mathcal{I}_{\theta}\right)\right) / G$ ] whose coarse moduli space is the Lawrence toric variety $X\left(\Sigma_{\theta}\right)=$ 
$\left(\mathbb{C}^{2 m} \backslash V\left(\mathcal{I}_{\theta}\right)\right) / G$. Let $Y \subset \mathbb{C}^{2 m} \backslash V\left(\mathcal{I}_{\theta}\right)$ be the subvariety defined by the ideal:

$$
I_{\beta^{\vee}}:=\left\langle\sum_{i=1}^{m}\left(\beta^{\vee}\right)^{\star}(x)_{i} a_{i j} z_{i} w_{i} \mid \forall x \in \operatorname{DG}(\beta)^{\star}\right\rangle,
$$

where $\left(\beta^{\vee}\right)^{\star}: \operatorname{DG}(\beta)^{\star} \rightarrow \mathbb{Z}^{m}$ is the dual map of $\beta^{\vee}$ and $\left(\beta^{\vee}\right)^{\star}(x)_{i}$ is the $i$ th component of the vector $\left(\beta^{\vee}\right)^{\star}(x)$. From [13], the hypertoric DM stack $\mathcal{M}(\mathcal{A})=[Y / G]$ whose coarse moduli space is the hypertoric variety $Y\left(\beta^{\vee}, \theta\right)=Y / G$.

Definition 3.1 [13]. The Lawrence toric DM stack is the toric DM stack $\mathcal{X}\left(\boldsymbol{\Sigma}_{\boldsymbol{\theta}}\right)$ corresponding to the Lawrence stacky fan $\boldsymbol{\Sigma}_{\boldsymbol{\theta}}$.

By [10], $X\left(\Sigma_{\theta}\right)$ is semi-projective. So the Lawrence toric DM stack $\mathcal{X}\left(\boldsymbol{\Sigma}_{\boldsymbol{\theta}}\right)$ is semi-projective by definition.

\subsection{Comparison of inertia stacks}

Next we compare the orbifold Chow ring of the hypertoric DM stack and the orbifold Chow ring of the Lawrence toric DM stack. First we compare the inertia stacks. From the map $\beta: \mathbb{Z}^{m} \rightarrow N$ which is given by vectors $\left\{b_{1}, \ldots, b_{m}\right\}$. Let Cone $(\beta)$ be a partially-ordered finite set of cones generated by $\bar{b}_{1}, \ldots, \bar{b}_{m}$. The partial order is defined by: $\sigma \prec \tau$ if $\sigma$ is a face of $\tau$, and we have the minimum element $\hat{0}$ which is the cone consisting of the origin. Let Cone $(\bar{N})$ be the set of all convex polyhedral cones in the lattice $\bar{N}$. Then we have a map

$$
C: \operatorname{Cone}(\beta) \longrightarrow \operatorname{Cone}(\bar{N})
$$

such that for any $\sigma \in \operatorname{Cone}(\beta), C(\sigma)$ is the cone in $\bar{N}$. Then $\Delta_{\beta}:=$ $(C$, Cone $(\beta))$ is a simplicial multi-fan in the sense of [9].

For the multi-fan $\Delta_{\boldsymbol{\beta}}$, let $\operatorname{Box}\left(\Delta_{\boldsymbol{\beta}}\right)$ be the set of pairs $(v, \sigma)$, where $\sigma$ is a cone in $\Delta_{\boldsymbol{\beta}}, v \in N$ such that $\bar{v}=\sum_{\rho_{i} \subseteq \sigma} \alpha_{i} b_{i}$ for $0<\alpha_{i}<1$. (Note that $\sigma$ is the minimal cone in $\Delta_{\boldsymbol{\beta}}$ satisfying the above condition.) From [13], an element $(v, \sigma) \in \operatorname{Box}\left(\Delta_{\boldsymbol{\beta}}\right)$ gives a component of the inertia stack $\mathcal{I}(\mathcal{M}(\mathcal{A}))$. Also consider the set $\operatorname{Box}\left(\boldsymbol{\Sigma}_{\boldsymbol{\theta}}\right)$ associated to the stacky fan $\boldsymbol{\Sigma}_{\boldsymbol{\theta}}$, see Section 2.2 for its definition. An element $v \in \operatorname{Box}\left(\boldsymbol{\Sigma}_{\boldsymbol{\theta}}\right)$ gives a component of the inertia stack $\mathcal{I}\left(\mathcal{X}\left(\boldsymbol{\Sigma}_{\boldsymbol{\theta}}\right)\right)$.

By the Lawrence lifting property, a vector $\bar{b}_{i}$ in $\bar{N}$ lifts to two vectors $\bar{b}_{\mathrm{L}, i}, \bar{b}_{\mathrm{L}, i}^{\prime}$ in $\bar{N}_{\mathrm{L}}$. Let $\left\{\bar{b}_{\mathrm{L}, i_{1}}, \ldots, \bar{b}_{\mathrm{L}, i_{k}}, \bar{b}_{\mathrm{L}, i_{1}}^{\prime}, \ldots, \bar{b}_{\mathrm{L}, i_{k}}^{\prime}\right\}$ be the Lawrence lifting of $\left\{\bar{b}_{i_{1}}, \ldots, \bar{b}_{i_{k}}\right\}$. 
Lemma 3.2. $\left\{\bar{b}_{i_{1}}, \ldots, \bar{b}_{i_{k}}\right\}$ generate a cone $\sigma$ in $\Delta_{\boldsymbol{\beta}}$ if and only if $\left\{\bar{b}_{\mathrm{L}, i_{1}}, \ldots\right.$, $\left.\bar{b}_{\mathrm{L}, i_{k}}, \bar{b}_{\mathrm{L}, i_{1}}^{\prime}, \ldots, \bar{b}_{\mathrm{L}, i_{k}}^{\prime}\right\}$ generate a cone $\sigma_{\theta}$ in $\Sigma_{\theta}$.

Proof. Suppose $\sigma$ is a cone in $\Delta_{\boldsymbol{\beta}}$ generated by $\left\{\bar{b}_{i_{1}}, \ldots, \bar{b}_{i_{k}}\right\}$, it is contained in a top-dimensional cone $\tau$. Assume that $\tau$ is generated by $\left\{\bar{b}_{i_{1}}, \ldots, \bar{b}_{i_{k}}\right.$, $\left.\bar{b}_{i_{k+1}}, \ldots, \bar{b}_{i_{d}}\right\}$. Let $C$ be the complement $\left\{\bar{b}_{1}, \ldots, \bar{b}_{m}\right\} \backslash \tau$. Then $C$ corresponds to a column basis of $\bar{\beta}^{\vee}$ in the map $\bar{\beta}^{\vee}: \mathbb{Z}^{m} \rightarrow \overline{\mathrm{DG}(\beta)}$. By the definition of $\Sigma_{\theta}$ in (3.4), $C$ corresponds to a maximal cone $\tau_{\theta}$ in $\Sigma_{\theta}$ which contains the rays generated by $\left\{\bar{b}_{\mathrm{L}, i_{1}}, \ldots, \bar{b}_{\mathrm{L}, i_{k}}, \bar{b}_{\mathrm{L}, i_{1}}^{\prime}, \ldots, \bar{b}_{\mathrm{L}, i_{k}}^{\prime}\right\}$. Thus these rays generate a cone $\sigma_{\theta}$ in $\Sigma_{\theta}$.

Conversely, suppose $\sigma_{\theta}$ is a cone in $\Sigma_{\theta}$ generated by $\left\{\bar{b}_{\mathrm{L}, i_{1}}, \ldots, \bar{b}_{\mathrm{L}, i_{k}}\right.$, $\left.\bar{b}_{\mathrm{L}, i_{1}}^{\prime}, \ldots, \bar{b}_{\mathrm{L}, i_{k}}^{\prime}\right\}$. Using the similar method above we prove that $\left\{\bar{b}_{i_{1}}, \ldots, \bar{b}_{i_{k}}\right\}$ must be contained in a top-dimensional cone of $\Delta_{\boldsymbol{\beta}}$. So $\left\{\bar{b}_{i_{1}}, \ldots, \bar{b}_{i_{k}}\right\}$ generate a cone $\sigma$ in $\Delta_{\beta}$.

Lemma 3.3. There is an one-to-one correspondence between the elements in $\operatorname{Box}\left(\boldsymbol{\Sigma}_{\boldsymbol{\theta}}\right)$ and the elements in $\operatorname{Box}\left(\boldsymbol{\Delta}_{\boldsymbol{\beta}}\right)$. Moreover, their degree shifting numbers coincide.

Proof. First the torsion elements in $\operatorname{Box}\left(\boldsymbol{\Sigma}_{\boldsymbol{\theta}}\right)$ and $\operatorname{Box}\left(\Delta_{\boldsymbol{\beta}}\right)$ are both isomorphic to $\mu=\operatorname{ker}(\alpha)=\operatorname{ker}\left(\alpha_{h}\right)$ in (2.3) and (3.5). Let $(v, \sigma) \in \operatorname{Box}\left(\Delta_{\beta}\right)$ with $\bar{v}=\sum_{\rho_{i} \subseteq \sigma} \alpha_{i} \bar{b}_{i}$. Then $v$ may be identified with an element (which we ambiguously denote by) $v \in G:=\operatorname{Hom}_{\mathbb{Z}}\left(\operatorname{DG}(\beta), \mathbb{C}^{*}\right)$. Certainly $v$ fixes a point in $\mathbb{C}^{m}$. Consider the map $\alpha$ in $(2.3)$, put $\alpha(v)=\left(\alpha^{1}(v), \ldots, \alpha^{m}(v)\right)$. Then $\alpha^{i}(v) \neq 1$ if $\rho_{i} \subseteq \sigma$, and $\alpha^{i}(v)=1$ otherwise. By Lemma 3.2, let $\left\{\bar{b}_{\mathrm{L}, i}, \bar{b}_{\mathrm{L}, i}^{\prime}: i=1, \ldots,|\sigma|\right\}$ be the Lawrence lifting of $\left\{\bar{b}_{i}\right\}_{\rho_{i} \subseteq \sigma}$. Since the action of $v$ on $\mathbb{C}^{2 m}$ is given by $\left(v, v^{-1}\right), v$ fixes a point in $\mathbb{C}^{2 m}$ and yields an element $v_{\theta}$ in $\operatorname{Box}\left(\boldsymbol{\Sigma}_{\boldsymbol{\theta}}\right)$. From the map (3.5), let

$$
\alpha_{h}\left(v_{\theta}\right)=\left(\alpha_{h}^{1}\left(v_{\theta}\right), \ldots, \alpha_{h}^{m}\left(v_{\theta}\right), \alpha_{h}^{m+1}\left(v_{\theta}\right), \cdots, \alpha_{h}^{2 m}\left(v_{\theta}\right)\right) .
$$

Then $\alpha_{h}^{i}\left(v_{\theta}\right) \neq 1$ and $\alpha_{h}^{i+m}\left(v_{\theta}\right) \neq 1$ if $\rho_{i} \subseteq \sigma ; \alpha_{h}^{i}\left(v_{\theta}\right)=\alpha_{h}^{i+m}\left(v_{\theta}\right)=1$ otherwise. So $\sigma_{\theta}\left(\bar{v}_{\theta}\right)=\left\{\bar{b}_{\mathrm{L}, i}, \bar{b}_{\mathrm{L}, i}^{\prime}: i=1, \ldots,|\sigma|\right\}$ is the minimal cone in $\Sigma_{\theta}$ containing $\bar{v}_{\theta}$. Furthermore, $\bar{v}_{\theta}=\sum_{\rho_{i} \subseteq \sigma} \alpha_{i} \bar{b}_{\mathrm{L}, i}+\sum_{\rho_{i} \subseteq \sigma}\left(1-\alpha_{i}\right) \bar{b}_{\mathrm{L}, i}^{\prime}$.

Conversely, given an element $v_{\theta} \in \operatorname{Box}\left(\boldsymbol{\Sigma}_{\boldsymbol{\theta}}\right)$, let $\sigma_{\theta}\left(\bar{v}_{\theta}\right)$ be the minimal cone in $\Sigma_{\theta}$ containing $\bar{v}_{\theta}$. Then from the action of $G$ on $\mathbb{C}^{2 m}$ and (3.7), we have $\alpha_{h}^{i}\left(v_{\theta}\right)=\left(\alpha_{h}^{i+m}\left(v_{\theta}\right)\right)^{-1}$. If $\alpha_{h}^{i}\left(v_{\theta}\right) \neq 1$, then $\alpha_{h}^{i+m}\left(v_{\theta}\right) \neq 1$, which means that $\bar{b}_{\mathrm{L}, i}, \bar{b}_{\mathrm{L}, i+m} \in \sigma_{\theta}\left(\bar{v}_{\theta}\right)$. The cone $\sigma_{\theta}\left(\bar{v}_{\theta}\right)$ is the one in $\Sigma_{\theta}$ containing $\bar{b}_{\mathrm{L}, i}, \bar{b}_{\mathrm{L}, i+m}$ satisfying this condition. Then $\bar{v}_{\theta}=\sum_{i}\left(\alpha_{i} \bar{b}_{\mathrm{L}, i}+\left(1-\alpha_{i}\right) \bar{b}_{\mathrm{L}, i}^{\prime}\right)$. By Lemma 3.2, $\sigma_{\theta}\left(\bar{v}_{\theta}\right)$ is the Lawrence lifting of a cone $\sigma$ generated by 
the $\left\{\bar{b}_{i}\right\}_{\mathrm{s}}$ in $\Delta_{\boldsymbol{\beta}}$. Let $v=\sum_{\rho_{i} \subseteq \sigma} \alpha_{i} b_{i}$. So it also determines an element $(v, \sigma) \in \operatorname{Box}\left(\Delta_{\beta}\right)$.

For $\left(v_{1}, \sigma_{1}\right),\left(v_{2}, \sigma_{2}\right),\left(v_{3}, \sigma_{3}\right) \in \operatorname{Box}\left(\Delta_{\boldsymbol{\beta}}\right)$, let $\sigma\left(\bar{v}_{1}, \bar{v}_{2}, \bar{v}_{3}\right)$ be the minimal cone containing $\bar{v}_{1}, \bar{v}_{2}, \bar{v}_{3}$ in $\Delta_{\beta}$ such that $\bar{v}_{1}+\bar{v}_{2}+\bar{v}_{3}=\sum_{\rho_{i} \subseteq \sigma\left(\bar{v}_{1}, \bar{v}_{2}, \bar{v}_{3}\right)}$ $a_{i} \bar{b}_{i}$ and $a_{i}=1,2$. Let $v_{\theta, 1}, v_{\theta, 2}, v_{\theta, 3}$ be the corresponding elements in $\operatorname{Box}\left(\boldsymbol{\Sigma}_{\boldsymbol{\theta}}\right)$ and $\sigma\left(\bar{v}_{\theta, 1}, \bar{v}_{\theta, 2}, \bar{v}_{\theta, 3}\right)$ the minimal cone containing $\bar{v}_{\theta, 1}, \bar{v}_{\theta, 2}, \bar{v}_{\theta, 3}$ in $\boldsymbol{\Sigma}_{\boldsymbol{\theta}}$. Then by Lemmas 3.2 and 3.3, $\sigma\left(\bar{v}_{\theta, 1}, \bar{v}_{\theta, 2}, \bar{v}_{\theta, 3}\right)$ is the Lawrence lifting of $\sigma\left(\bar{v}_{1}, \bar{v}_{2}, \bar{v}_{3}\right)$. Suppose that $\sigma$ is generated by $\left\{\bar{b}_{i_{1}}, \ldots, \bar{b}_{i_{s}}\right\}$, then $\sigma\left(\bar{v}_{\theta, 1}, \bar{v}_{\theta, 2}, \bar{v}_{\theta, 3}\right)$ is generated by $\left\{\bar{b}_{\mathrm{L}, i_{1}}, \ldots, \bar{b}_{\mathrm{L}, i_{s}}, \bar{b}_{\mathrm{L}, i_{1}}^{\prime}, \ldots, \bar{b}_{\mathrm{L}, i_{s}}^{\prime}\right\}$, the Lawrence lifting of $\left\{\bar{b}_{i_{1}}, \ldots, \bar{b}_{i_{s}}\right\}$. Let $\left\{\bar{b}_{j_{1}}, \ldots, \bar{b}_{j_{m-l-s}}\right\}$ be the rays not in $\sigma \cup \operatorname{link}(\sigma)$, we have the Lawrence lifting $\left\{\bar{b}_{\mathrm{L}, j_{1}}, \ldots, \bar{b}_{\mathrm{L}, j_{m-l-s}}, \bar{b}_{\mathrm{L}, j_{1}}^{\prime}, \ldots\right.$, $\left.\bar{b}_{\mathrm{L}, j_{m-l-s}}^{\prime}\right\}$. Then from the definition of Lawrence fan $\Sigma_{\theta}$ in (3.4), we have the following lemma.

Lemma 3.4. There exist $m-l-s$ vectors in $\left\{\bar{b}_{\mathrm{L}, j_{1}}, \ldots, \bar{b}_{\mathrm{L}, j_{m-l-s}}, \bar{b}_{\mathrm{L}, j_{1}}^{\prime}, \ldots\right.$, $\left.\bar{b}_{\mathrm{L}, j_{m-l-s}}^{\prime}\right\}$ such that the rays they generate plus the rays in $\sigma\left(\bar{v}_{\theta, 1}, \bar{v}_{\theta, 2}, \bar{v}_{\theta, 3}\right)$ generate a cone $\sigma_{\theta}$ in $\Sigma_{\theta}$.

Proposition 3.5. The stack $\mathcal{X}\left(\boldsymbol{\Sigma}_{\boldsymbol{\theta}} / \sigma_{\theta}\right)$ is also a Lawrence toric DM stack.

Proof. For simplicity, put $\sigma:=\sigma\left(\bar{v}_{1}, \bar{v}_{2}, \bar{v}_{3}\right)$. Suppose there are $l$ rays in the $\operatorname{link}(\sigma)$. Then by Lemma 3.2 there are $2 l$ rays in $\operatorname{link}\left(\sigma_{\theta}\right)$, the Lawrence lifting of $\operatorname{link}(\sigma)$. Let $s:=|\sigma|$, then $2 s+m-l-s=\left|\sigma_{\theta}\right|$. Applying Gale dual to the diagrams:

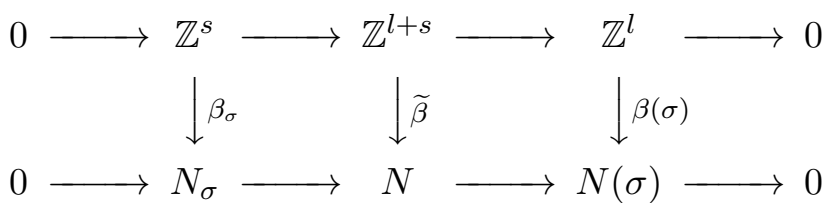

and

yields

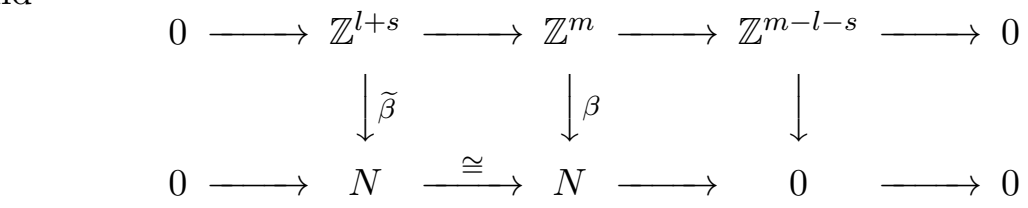


and

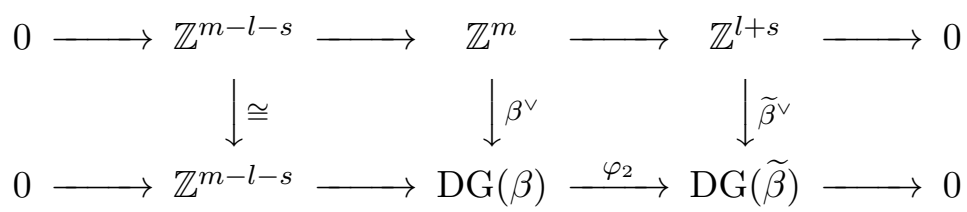

Since $\mathbb{Z}^{s} \cong N_{\sigma}$, we have $\operatorname{DG}\left(\beta_{\sigma}\right)=0$. We add two exact sequences

$$
0 \longrightarrow \mathbb{Z}^{l} \longrightarrow \mathbb{Z}^{m} \longrightarrow \mathbb{Z}^{m-l} \longrightarrow 0
$$

and

$$
0 \longrightarrow 0 \longrightarrow \mathbb{Z}^{m} \longrightarrow \mathbb{Z}^{m} \longrightarrow 0
$$

on the rows of the diagrams (3.8), (3.9) and make suitable maps to the Gale duals we get

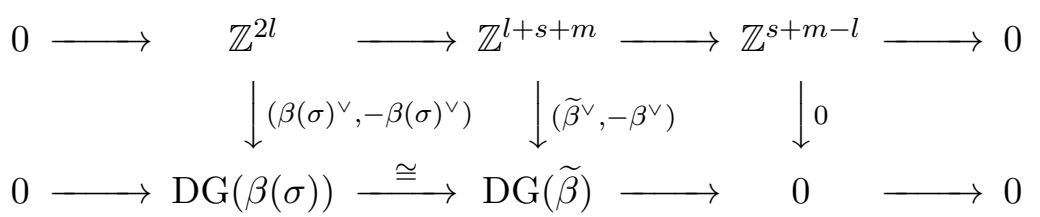

and

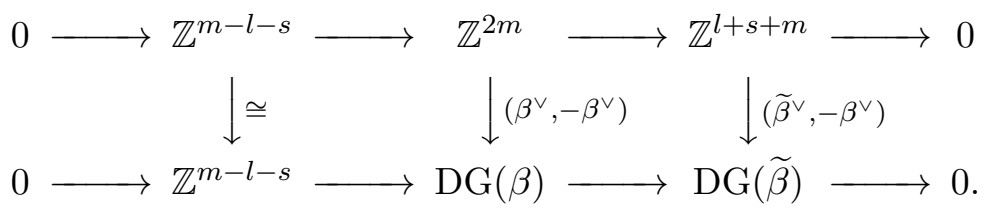

Applying Gale dual to (3.10), (3.11) we get

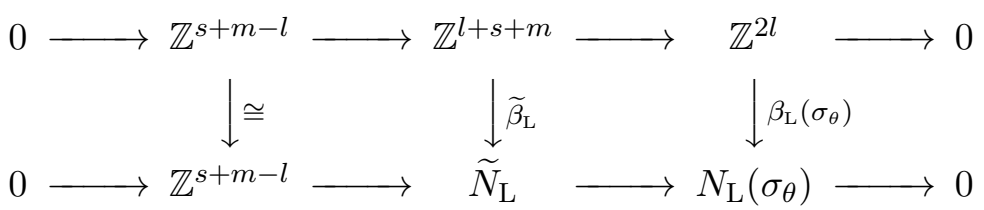

and

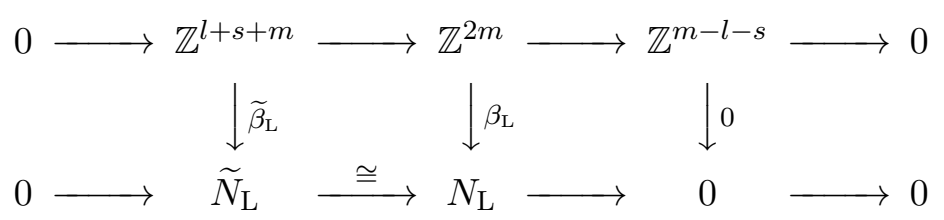


For the generic element $\theta$, from the map $\varphi_{2}$ in $(3.9), \theta$ induces $\tilde{\theta} \in \operatorname{DG}(\widetilde{\beta})$, and from the isomorphism $\varphi_{1}$ in $(3.8), \widetilde{\theta}=\theta(\sigma) \in \operatorname{DG}(\beta(\sigma))$. So we a quotient stacky hyperplane arrangement $\mathcal{A}(\sigma)=(N(\sigma), \beta(\sigma), \theta(\sigma))$. From the above diagrams, we see that the quotient fan $\Sigma_{\theta} / \sigma_{\theta}$ in $\bar{N}_{\mathrm{L}}\left(\sigma_{\theta}\right)$ also comes from a Lawrence construction of the map $\beta(\sigma)^{\vee}: \mathbb{Z}^{l} \rightarrow \operatorname{DG}(\beta(\sigma))$. Let $X(\sigma)=\mathbb{C}^{2 l} \backslash V\left(\mathcal{I}_{\theta(\sigma)}\right)$, where $\mathcal{I}_{\theta(\sigma)}$ is the irrelevant ideal of the quotient fan $\Sigma_{\theta} / \sigma_{\theta}$. Let $G(\sigma)=\operatorname{Hom}_{\mathbb{Z}}\left(\operatorname{DG}(\beta(\sigma)), \mathbb{C}^{*}\right)$. The stack $\mathcal{X}\left(\boldsymbol{\Sigma}_{\boldsymbol{\theta}} / \sigma_{\theta}\right)=$ $[X(\sigma) / G(\sigma)]$ is a Lawrence toric Deligne-Mumford stack.

Corollary 3.6. $\mathcal{M}\left(\mathcal{A}\left(\sigma\left(\bar{v}_{1}, \bar{v}_{2}, \bar{v}_{3}\right)\right)\right)$ is the hypertoric DM stack associated to the quotient Lawrence toric DM stack $\mathcal{X}\left(\boldsymbol{\Sigma}_{\boldsymbol{\theta}} / \sigma_{\theta}\right)$.

Proof. $\mathcal{M}\left(\mathcal{A}\left(\sigma\left(\bar{v}_{1}, \bar{v}_{2}, \bar{v}_{3}\right)\right)\right)$ is constructed in [13] as a quotient stack $[Y(\sigma) /$ $G(\sigma)]$, where $Y(\sigma) \subset X(\sigma)$ is defined by $I_{\beta(\sigma)^{\vee}}$, which is the ideal in (3.6) corresponding to the map $\beta(\sigma)^{\vee}$ in (3.8). So the stack $\mathcal{M}\left(\mathcal{A}\left(\sigma\left(\bar{v}_{1}, \bar{v}_{2}, \bar{v}_{3}\right)\right)\right)$ is the associated hypertoric DM stack in the Lawrence toric DM stack $\mathcal{X}\left(\boldsymbol{\Sigma}_{\boldsymbol{\theta}} / \sigma_{\theta}\right)$.

Remark 3.7. For any $v_{\theta} \in \operatorname{Box}\left(\boldsymbol{\Sigma}_{\boldsymbol{\theta}}\right)$, let $v_{\theta}^{-1}$ be its inverse. We have the quotient Lawrence toric stack $\mathcal{X}\left(\boldsymbol{\Sigma}_{\boldsymbol{\theta}} / \sigma_{\theta}\right)$. Let $(v, \sigma)$ be the corresponding element in $\operatorname{Box}\left(\Delta_{\beta}\right)$, then

$$
\mathcal{M}\left(\mathcal{A}\left(\sigma\left(\bar{v}, \bar{v}^{-1}, 1\right)\right)\right) \cong \mathcal{M}(\mathcal{A}(\sigma))
$$

By Proposition 3.5 and Corollary 3.6, the twisted sector $\mathcal{M}(\mathcal{A}(\sigma))$ is the associated hypertoric DM stack of the Lawrence toric DM stack $\mathcal{X}\left(\boldsymbol{\Sigma}_{\boldsymbol{\theta}} / \sigma_{\theta}\right)$.

Remark 3.8. From Lemma 3.4, the cone $\sigma_{\theta}$ is not the minimal cone $\sigma\left(\bar{v}_{\theta, 1}, \bar{v}_{\theta, 2}, \bar{v}_{\theta, 3}\right)$ containing $\bar{v}_{\theta, 1}, \bar{v}_{\theta, 2}, \bar{v}_{\theta, 3}$ in $\Sigma_{\theta}$. So $\mathcal{X}\left(\Sigma_{\theta} / \sigma\left(\bar{v}_{\theta, 1}, \bar{v}_{\theta, 2}, \bar{v}_{\theta, 3}\right)\right)$ is not a Lawrence toric DM stack. But from the construction of Lawrence toric DM stack, the quotient stack $\mathcal{X}\left(\Sigma_{\theta} / \sigma\left(\bar{v}_{\theta, 1}, \bar{v}_{\theta, 2}, \bar{v}_{\theta, 3}\right)\right)$ is homotopy equivalent to the quotient stack $\mathcal{X}\left(\Sigma_{\theta} / \sigma_{\theta}\right)$. Since we do not need this to compare the orbifold Chow ring, we omit the details.

\subsection{Comparison of orbifold Chow rings}

Recall that $N_{\mathrm{L}}=\bar{N}_{\mathrm{L}} \oplus N_{\mathrm{L} \text {,tor }}$, where $N_{\mathrm{L} \text {,tor }}$ is the torsion subgroup of $N_{\mathrm{L}}$. Let $N_{\Sigma_{\theta}}=N_{\mathrm{L}, \text { tor }} \oplus\left|\Sigma_{\theta}\right|$. By Theorem 1.1, we have 
Proposition 3.9. The orbifold Chow ring $A_{\text {orb }}^{*}\left(\mathcal{X}\left(\boldsymbol{\Sigma}_{\theta}\right)\right)$ of the Lawrence toric DM stack $\mathcal{X}\left(\boldsymbol{\Sigma}_{\boldsymbol{\theta}}\right)$ is isomorphic to the ring

$$
\frac{\mathbb{Q}\left[N_{\Sigma_{\theta}}\right]}{\left\{\sum_{i=1}^{m} e\left(b_{\mathrm{L}, i}\right) y^{b_{L, i}}+\sum_{i=1}^{m} e\left(b_{\mathrm{L}, i}^{\prime}\right) y^{b_{\mathrm{L}, i}^{\prime}}: e \in N_{\mathrm{L}}^{\star}\right\}} .
$$

Recall in [13] that for any $c \in N$, there is a cone $\sigma \in \Delta_{\boldsymbol{\beta}}$ such that $\bar{c}=\sum_{\rho_{i} \subseteq \sigma} \alpha_{i} \bar{b}_{i}$ where $\alpha_{i}>0$ are rational numbers. Let $N^{\Delta_{\beta}}$ denote all the pairs $(c, \sigma)$. Then $N^{\Delta_{\beta}}$ gives rise a group ring

$$
\mathbb{Q}\left[\Delta_{\boldsymbol{\beta}}\right]=\bigoplus_{(c, \sigma) \in N^{\Delta_{\boldsymbol{\beta}}}} \mathbb{Q} \cdot y^{(c, \sigma)}
$$

where $y$ is a formal variable. For any $(c, \sigma) \in N^{\Delta_{\beta}}$, there exists a unique element $(v, \tau) \in \operatorname{Box}\left(\Delta_{\boldsymbol{\beta}}\right)$ such that $\tau \subseteq \sigma$ and $c=v+\sum_{\rho_{i} \subseteq \sigma} m_{i} b_{i}$, where $m_{i}$ are non-negative integers. We call $(v, \tau)$ the fractional part of $(v, \sigma)$. We define the ceiling function for fans. For $(c, \sigma)$, define the ceiling function $\lceil c\rceil_{\sigma}$ by $\lceil c\rceil_{\sigma}=\sum_{\rho_{i} \subseteq \tau} b_{i}+\sum_{\rho_{i} \subseteq \sigma} m_{i} b_{i}$. Note that if $\bar{v}=0,\lceil c\rceil_{\sigma}=\sum_{\rho_{i} \subseteq \sigma} m_{i} b_{i}$. For two pairs $\left(c_{1}, \sigma_{1}\right),\left(c_{2}, \sigma_{2}\right)$, if $\sigma_{1} \cup \sigma_{2}$ is a cone in $\Delta_{\boldsymbol{\beta}}$, define $\epsilon\left(c_{1}, c_{2}\right):=$ $\left\lceil c_{1}\right\rceil_{\sigma_{1}}+\left\lceil c_{2}\right\rceil_{\sigma_{2}}-\left\lceil c_{1}+c_{2}\right\rceil_{\sigma_{1} \cup \sigma_{2}}$. Let $\sigma_{\epsilon} \subseteq \sigma_{1} \cup \sigma_{2}$ be the minimal cone in $\Delta_{\boldsymbol{\beta}}$ containing $\epsilon\left(c_{1}, c_{2}\right)$ such that $\left(\epsilon\left(c_{1}, c_{2}\right), \sigma_{\epsilon}\right) \in N^{\Delta_{\boldsymbol{\beta}}}$. We define the grading on $\mathbb{Q}\left[\Delta_{\boldsymbol{\beta}}\right]$ as follows. For any $(c, \sigma)$, write $c=v+\sum_{\rho_{i} \subseteq \sigma} m_{i} b_{i}$, then $\operatorname{deg}\left(y^{(c, \sigma)}\right)=|\tau|+\sum_{\rho_{i} \subseteq \sigma} m_{i}$, where $|\tau|$ is the dimension of $\tau$. By abuse of notation, we write $y^{\left(b_{i}, \rho_{i}\right)}$ as $y^{b_{i}}$. The multiplication is defined by

$y^{\left(c_{1}, \sigma_{1}\right)} \cdot y^{\left(c_{2}, \sigma_{2}\right)}:= \begin{cases}(-1)^{\left|\sigma_{\epsilon}\right|} y^{\left(c_{1}+c_{2}+\epsilon\left(c_{1}, c_{2}\right), \sigma_{1} \cup \sigma_{2}\right)} & \text { if } \sigma_{1} \cup \sigma_{2} \text { is a cone in } \Delta_{\boldsymbol{\beta}}, \\ 0 & \text { otherwise }\end{cases}$

From the property of ceiling function, we check that the multiplication is commutative and associative. So $\mathbb{Q}\left[\Delta_{\beta}\right]$ is a unital associative commutative ring. In [13], it is shown that

$$
A_{\mathrm{orb}}^{*}(\mathcal{M}(\mathcal{A})) \cong \frac{\mathbb{Q}\left[\Delta_{\boldsymbol{\beta}}\right]}{\left\{\sum_{i=1}^{m} e\left(b_{i}\right) y^{b_{i}}: e \in N^{\star}\right\}} .
$$

Consider the map $\beta: \mathbb{Z}^{m} \rightarrow N$ which is given by the vectors $\left\{b_{1}, \ldots, b_{m}\right\}$. We take $\{1, \ldots, m\}$ as the vertex set of the matroid complex $M_{\beta}$, defined from $\beta$ by requiring that $F \in M_{\beta}$ iff the vectors $\left\{\bar{b}_{i}\right\}_{i \in F}$ are linearly independent in $\bar{N}$. A face $F \in M_{\beta}$ corresponds to a cone in $\Delta_{\beta}$ generated by 
$\left\{\bar{b}_{i}\right\}_{i \in F}$. By [16], the "Stanley-Reisner" ring of the matroid $M_{\beta}$ is

$$
\mathbb{Q}\left[M_{\beta}\right]=\frac{\mathbb{Q}\left[y^{b_{1}}, \ldots, y^{b_{m}}\right]}{I_{M_{\beta}}},
$$

where $I_{M_{\beta}}$ is the matroid ideal generated by the set of square-free monomials

$$
\left\{y^{b_{i_{1}}} \cdots y^{b_{i_{k}}} \mid \bar{b}_{i_{1}}, \ldots, \bar{b}_{i_{k}} \text { linearly dependent in } \bar{N}\right\}
$$

It is proved in [13] that,

$$
\mathbb{Q}\left[\Delta_{\boldsymbol{\beta}}\right] \cong \bigoplus_{(v, \sigma) \in \operatorname{Box}\left(\Delta_{\boldsymbol{\beta}}\right)} y^{(v, \sigma)} \cdot \mathbb{Q}\left[M_{\beta}\right]
$$

For any $\left(v_{1}, \sigma_{1}\right),\left(v_{2}, \sigma_{2}\right) \in \operatorname{Box}\left(\Delta_{\beta}\right)$, let $\left(v_{3}, \sigma_{3}\right)$ be the unique element in $\operatorname{Box}\left(\Delta_{\boldsymbol{\beta}}\right)$ such that $v_{1}+v_{2}+v_{3} \equiv 0$ in the local group given by $\sigma_{1} \cup \sigma_{2}$, where $\equiv 0$ means that there exists a cone $\sigma\left(\bar{v}_{1}, \bar{v}_{2}, \bar{v}_{3}\right)$ in $\Delta_{\beta}$ such that $\bar{v}_{1}+\bar{v}_{2}+\bar{v}_{3}=\sum_{\rho_{i} \subseteq \sigma\left(\bar{v}_{1}, \bar{v}_{2}, \bar{v}_{3}\right)} a_{i} \bar{b}_{i}$, where $a_{i}=1$ or 2 . Let $\bar{v}_{1}=\sum_{\rho_{j} \subseteq \sigma_{1}} \alpha_{j}^{1} \bar{b}_{j}$, $\bar{v}_{2}=\sum_{\rho_{j} \subseteq \sigma_{2}} \alpha_{j}^{2} \bar{b}_{j}, \bar{v}_{3}=\sum_{\rho_{j} \subseteq \sigma_{3}} \alpha_{j}^{3} \bar{b}_{j}$ with $0<\alpha_{j}^{1}, \alpha_{j}^{2}, \alpha_{j}^{3}<1$. Let $I$ be the set of $i$ such that $a_{i}=1$ and $\alpha_{j}^{1}, \alpha_{j}^{2}, \alpha_{j}^{3}$ exist, $J$ the set of $j$ such that $\rho_{j}$ belongs to $\sigma\left(\bar{v}_{1}, \bar{v}_{2}, \bar{v}_{3}\right)$ but not $\sigma_{3}$. If $(v, \sigma) \in \operatorname{Box}\left(\Delta_{\beta}\right)$, let $(\check{v}, \sigma)$ be the inverse of $(v, \sigma)$. Except torsion elements, equivalently, if $\bar{v}=\sum_{\rho_{i} \subseteq \sigma} \alpha_{i} \bar{b}_{i}$ for $0<\alpha_{i}<1$, then $\check{\bar{v}}=\sum_{\rho_{i} \subseteq \sigma}\left(1-\alpha_{i}\right) \bar{b}_{i}$. By abuse of notation, we write $y^{\left(b_{i}, \rho_{i}\right)}$ as $y^{b_{i}}$. We have $v_{1}+v_{2}=\check{v}_{3}+\sum_{a_{i}=2} b_{i}+\sum_{j \in J} b_{j}$. From (3.13), [13, Lemma 5.11] and [13, Lemma 5.12], if $\bar{v}_{1}, \bar{v}_{2} \neq 0$, we have

$$
\left\lceil v_{1}\right\rceil_{\sigma_{1}}+\left\lceil v_{2}\right\rceil_{\sigma_{2}}-\left\lceil v_{1}+v_{2}\right\rceil_{\sigma_{1} \cup \sigma_{2}}= \begin{cases}\sum_{i \in I} b_{i}+\sum_{j \in J} b_{j} & \text { if } \bar{v}_{1} \neq \check{\bar{v}}_{2}, \\ \sum_{j \in J} b_{j} & \text { if } \bar{v}_{1}=\bar{v}_{2}\end{cases}
$$

So it is easy to check that the multiplication $y^{\left(v_{1}, \sigma_{1}\right)} \cdot y^{\left(v_{2}, \sigma_{2}\right)}$ can be written as

$$
\begin{cases}(-1)^{|I|+|J|} y^{\left(\check{v}_{3}, \sigma_{3}\right)} \cdot \prod_{a_{i}=2} y^{b_{i}} \cdot & \\ \prod_{i \in I} y^{b_{i}} \cdot \prod_{j \in J} y^{2 b_{j}} & \text { if } \bar{v}_{1}, \bar{v}_{2} \in \sigma \text { for } \sigma \in \Delta_{\boldsymbol{\beta}} \text { and } \bar{v}_{1} \neq \check{\bar{v}}_{2}, \\ (-1)^{|J|} \prod_{j \in J} y^{2 b_{j}} & \text { if } \bar{v}_{1}, \bar{v}_{2} \in \sigma \text { for } \sigma \in \Delta_{\boldsymbol{\beta}} \text { and } \bar{v}_{1}=\check{\bar{v}}_{2}, \\ 0 & \text { otherwise. }\end{cases}
$$

The following is the main result of this section. 
Theorem 3.10. There is an isomorphism of orbifold Chow rings $A_{\mathrm{orb}}^{*}$ $\left(\mathcal{X}\left(\boldsymbol{\Sigma}_{\boldsymbol{\theta}}\right)\right) \cong A_{\text {orb }}^{*}(\mathcal{M}(\mathcal{A}))$.

Proof. The ring $\mathbb{Q}\left[N_{\Sigma_{\theta}}\right]$ is generated by $\left\{y^{b_{\mathrm{L}, i}}, y^{b_{\mathrm{L}, i}^{\prime}}: i=1, \ldots, m\right\}$ and $y^{v_{\theta}}$ for $v_{\theta} \in \operatorname{Box}\left(\boldsymbol{\Sigma}_{\boldsymbol{\theta}}\right)$ by the definition. By Lemma 3.3, define a morphism

$$
\phi: \mathbb{Q}\left[N_{\Sigma_{\theta}}\right] \rightarrow \mathbb{Q}\left[\Delta_{\beta}\right]
$$

by $y^{b_{\mathrm{L}, i}} \mapsto y^{b_{i}}, y^{b_{\mathrm{L}, i}^{\prime}} \mapsto-y^{b_{i}}$ and $y^{v_{\theta}} \mapsto y^{(v, \sigma)}$. By [10], the ideal $\mathcal{I}_{\theta}$ goes to the ideal $I_{M_{\beta}}$ and the relation $\left\{\sum_{i=1}^{m} e\left(b_{\mathrm{L}, i}\right) y^{b_{\mathrm{L}, i}}+\sum_{i=1}^{m} e\left(b_{\mathrm{L}, i}^{\prime}\right) y^{b_{\mathrm{L}, i}^{\prime}}: e \in N_{\mathrm{L}}^{\star}\right\}$ goes to the relation $\left\{\sum_{i=1}^{m} e\left(b_{i}\right) y^{b_{i}}: e \in N^{\star}\right\}$. Thus the two rings are isomorphic as modules.

It remains to check the multiplications. For any $y^{v_{\theta}}$ and $y^{b_{L, i}}$ or $y^{b_{L, i}^{\prime}}$, let $y^{(v, \sigma)}$ be the corresponding element in $\mathbb{Q}\left[\Delta_{\beta}\right]$. By the property of $v_{\theta}$ and Lemma 3.3, the minimal cone in $\Sigma_{\theta}$ containing $\bar{v}_{\theta}, \bar{b}_{\mathrm{L}, i}$ must contains $\bar{b}_{L, i}^{\prime}$. By Lemma 3.2 , there is a cone in $\Delta_{\boldsymbol{\beta}}$ containing $\bar{v}, \bar{b}_{i}$. In this way, $y^{v_{\theta}} \cdot y^{b_{\mathrm{L}, i}}$ goes to $y^{(v, \sigma)} \cdot y^{b_{i}}$ and $y^{v_{\theta}} \cdot y^{b_{\mathrm{L}, i}^{\prime}}$ goes to $-y^{(v, \sigma)} \cdot y^{b_{i}}$. If there is no cone in $\Sigma_{\theta}$ containing $\bar{v}_{\theta}, \bar{b}_{\mathrm{L}, i}, \bar{b}_{\mathrm{L}, i}^{\prime}$, then by Lemma 3.2 there is no cone in $\Delta_{\boldsymbol{\beta}}$ containing $\bar{v}, \bar{b}_{i}$. So $y^{v_{\theta}} \cdot y^{b_{\mathrm{L}, i}}=0$ goes to $y^{(v, \sigma)} \cdot y^{b_{i}}=0$ and $y^{v_{\theta}} \cdot y^{b_{\mathrm{L}, i}^{\prime}}=0$ goes to $-y^{(v, \sigma)} \cdot y^{b_{i}}=0$.

For any $y^{v_{\theta, 1}}, y^{v_{\theta, 2}}$, let $y^{\left(v_{1}, \sigma_{1}\right)}, y^{\left(v_{2}, \sigma_{2}\right)}$ be the corresponding elements in $\mathbb{Q}\left[\Delta_{\boldsymbol{\beta}}\right]$. If there is no cone in $\Sigma_{\theta}$ containing $\bar{v}_{\theta, 1}, \bar{v}_{\theta, 2}$, then by Lemmas 3.2 and 3.3 , there is no cone in $\Delta_{\beta}$ containing $\bar{v}_{1}, \bar{v}_{2}$. So $y^{v_{\theta, 1}} \cdot y^{v_{\theta, 2}}=0$ goes to $y^{\left(v_{1}, \sigma_{1}\right)} \cdot y^{\left(v_{2}, \sigma_{2}\right)}=0$. Suppose there is a cone containing $\bar{v}_{\theta, 1}, \bar{v}_{\theta, 2}$, let $v_{\theta, 3} \in \operatorname{Box}\left(\boldsymbol{\Sigma}_{\theta}\right)$ such that $v_{\theta, 1}+v_{\theta, 2}+v_{\theta, 3} \equiv 0$. Let $\sigma\left(\bar{v}_{\theta, 1}, \bar{v}_{\theta, 2}, \bar{v}_{\theta, 3}\right)$ be the minimal cone containing $\bar{v}_{\theta, 1}, \bar{v}_{\theta, 2}, \bar{v}_{\theta, 3}$ in $\Sigma_{\theta}$. Then by Lemmas 3.2 and 3.3, $\sigma\left(\bar{v}_{\theta, 1}, \bar{v}_{\theta, 2}, \bar{v}_{\theta, 3}\right)$ is the Lawrence lifting of $\sigma\left(\bar{v}_{1}, \bar{v}_{2}, \bar{v}_{3}\right)$ for $\left(v_{1}, \sigma_{1}\right),\left(v_{2}, \sigma_{2}\right),\left(v_{3}, \sigma_{3}\right) \in \operatorname{Box}\left(\Delta_{\beta}\right)$. So we may write $\bar{v}_{\theta, 1}+\bar{v}_{\theta, 2}+\bar{v}_{\theta, 3}=$ $\sum_{\rho_{i} \subseteq \sigma\left(\bar{v}_{1}, \bar{v}_{2}, \bar{v}_{3}\right)} a_{i} \bar{b}_{\mathrm{L}, i}+\sum_{\rho_{i} \subseteq \sigma\left(\bar{v}_{1}, \bar{v}_{2}, \bar{v}_{3}\right)} a_{i}^{\prime} \bar{b}_{\mathrm{L}, i}^{\prime}$. The corresponding $\bar{v}_{1}+\bar{v}_{2}+\bar{v}_{3}$ $=\sum_{\rho_{i} \subseteq \sigma\left(\bar{v}_{1}, \bar{v}_{2}, \bar{v}_{3}\right)} a_{i} \bar{b}_{i}$. Let $(\tilde{v}, \sigma)$ be the inverse of $(v, \sigma)$ in $\operatorname{Box}\left(\Delta_{\boldsymbol{\beta}}\right)$, i.e., if $v$ is nontorsion and $\bar{v}=\sum_{\rho_{i} \subseteq \sigma} \alpha_{i} \bar{b}_{i}$ for $0<\alpha_{i}<1$, then $\check{\bar{v}}=\sum_{\rho_{i} \subseteq \sigma}$ $\left(1-\alpha_{i}\right) \bar{b}_{i}$. The $\check{v}_{\theta}$ is defined similarly in $\operatorname{Box}\left(\boldsymbol{\Sigma}_{\boldsymbol{\theta}}\right)$. The notation $J$ represents the set of $j$ such that $\rho_{j}$ belongs to $\sigma\left(\bar{v}_{1}, \bar{v}_{2}, \bar{v}_{3}\right)$ but not $\sigma_{3}$, the corresponding $\rho_{L, j}, \rho_{L, j}^{\prime}$ belong to $\sigma\left(\bar{v}_{\theta, 1}, \bar{v}_{\theta, 2}, \bar{v}_{\theta, 3}\right)$ but not $\sigma\left(\bar{v}_{\theta, 3}\right)$.

If some $\bar{v}_{\theta, i}=0$ which means that $v_{\theta, i}$ is a torsion. Then from Lemma 3.3 the corresponding $v$ is also a torsion element. In this case, we know that the orbifold cup product $y^{v_{\theta, 1}} \cdot y^{v_{\theta, 2}}$ is the usual product, and under the map $\phi$, is equal to $y^{\left(v_{1}, \sigma_{1}\right)} \cdot y^{\left(v_{2}, \sigma_{2}\right)}$. 
If $\bar{v}_{\theta, 1}=\check{\bar{v}}_{\theta, 2}$, then $\bar{v}_{\theta, 3}=0$ and the obstruction bundle over the corresponding 3 -twisted sector is zero. The set $J$ is the set $j$ such that $\rho_{j}$ belongs to $\sigma\left(\bar{v}_{\theta, 1}\right)$. So from [3], we have

$$
y^{v_{\theta, 1}} \cdot y^{v_{\theta, 2}}=\prod_{j \in J} y^{b_{\mathrm{L}, j}} \cdot y^{b_{\mathrm{L}, j}^{\prime}}
$$

Under the map $\phi$, we see that $y^{\left(v_{1}, \sigma_{1}\right)} \cdot y^{\left(v_{2}, \sigma_{2}\right)}$ is equal to the second line in the product $(3.15)$.

If $\bar{v}_{\theta, 1} \neq \bar{v}_{\theta, 2}$, then $\bar{v}_{\theta, 3} \neq 0$ and the obstruction bundle is given by Proposition 2.10. If all $\alpha_{j}^{1}, \alpha_{j}^{2}, \alpha_{j}^{3}$ exist, the coefficients $a_{i}$ and $a_{i}^{\prime}$ satisfy that if $a_{i}=1$ then $a_{i}^{\prime}=2$, and if $a_{i}=2$ then $a_{i}^{\prime}=1$. So from [3],

$$
y^{v_{\theta, 1}} \cdot y^{v_{\theta, 2}}=y^{\check{v}_{\theta, 3}} \cdot \prod_{a_{i}=2} y^{b_{\mathrm{L}, i}} \cdot \prod_{i \in I} y^{b_{\mathrm{L}, i}^{\prime}} \cdot \prod_{j \in J} y^{b_{\mathrm{L}, j}} \cdot y^{b_{\mathrm{L}, j}^{\prime}}
$$

Under the map $\phi$, we see that $y^{\left(v_{1}, \sigma_{1}\right)} \cdot y^{\left(v_{2}, \sigma_{2}\right)}$ is equal to the first line in the product (3.15). By Lemma 3.3, the box elements have the same orbifold degrees. By Corollary 3.6 and the definition of orbifold cup product in (2.7), the products $y^{v_{\theta, 1}} \cdot y^{v_{\theta, 2}}$ and $y^{\left(v_{1}, \sigma_{1}\right)} \cdot y^{\left(v_{2}, \sigma_{2}\right)}$ have the same degrees in both Chow rings. So $\phi$ induces a ring isomorphism $A_{\text {orb }}^{*}\left(\mathcal{X}\left(\boldsymbol{\Sigma}_{\boldsymbol{\theta}}\right)\right) \cong$ $A_{\text {orb }}^{*}(\mathcal{M}(\mathcal{A}))$.

Remark 3.11. The presentation (3.14) of orbifold Chow ring only depends on the matroid complex corresponding to the $\operatorname{map} \beta: \mathbb{Z}^{m} \rightarrow N$, not $\theta$. Note that the presentation (3.12) depends on the fan $\Sigma_{\theta}$. We could not see explicitly from this presentation that the ring is independent to the choice of generic elements $\theta$.

\section{Acknowledgments}

We thank Kai Behrend and Nicholas Proudfoot for valuable discussions.

\section{References}

[1] D. Abramovich, T. Graber and A. Vistoli, Algebraic orbifold quantum product, Orbifolds in mathematics and physics (Madison,WI, 2001), Contemporary Mathematics, 310, American Mathematic Society, providence, RI, 2002, 1-24, math.AG/0112004. 
[2] R. Bielawski and A. Dancer, The geometry and topology of toric hyperkähler manifolds, Comm. Anal. Geom. 8(4), (2000), 727-760.

[3] L. Borisov, L. Chen and G. Smith, The orbifold Chow ring of toric Deligne-Mumford stacks, J. Amer. Math. Soc. 18(1), (2005), 193-215, math.AG/0309229.

[4] B. Chen and S. Hu, A deRham model for Chen-Ruan cohomology ring of abelian orbifolds, Math. Ann. 336(1), (2006), 51-71, math.SG/ 0408265.

[5] W. Chen and Y. Ruan, A new cohomology theory for orbifolds, Comm. Math. Phys. 248(1), (2004), 1-31, math.AG/0004129.

[6] W. Chen and Y. Ruan, Orbifold Gromov-Witten theory, Orbifolds in mathematics and physics (Madison, WI, 2001), Contemporary Mathematical, 310, American Mathematical Society, 2002, 25-85, math.AG/0103156.

[7] D. Cox, The homogeneous coordinate ring of a toric variety, J. Algebraic Geom. 4 (1995), 17-50.

[8] W. Fulton, Introduction to toric varieties, Annals of Mathematics Studies, 131, Princeton University Press, Princeton, NJ, 1993.

[9] A. Hattori and M. Masuda, Theory of multi-fans, Osaka J. Math. 40 (2003), 1-68.

[10] T. Hausel and B. Sturmfels, Toric hyperkähler varieties, Doc. Math. 7 (2002), 495-534.

[11] T. Jarvis, R. Kaufmann and T. Kimura, Stringy K-theory and the Chern character, Invent. Math. 168(1), (2007), 23-81, math.AG/0502280.

[12] Y. Jiang, The orbifold cohomology ring of simplicial toric stack bundles, Illinois J. Math., to appear, math.AG/0504563.

[13] Y. Jiang and H.-H. Tseng, The orbifold Chow ring of hypertoric Deligne-Mumford stacks, J. Reine Angew. Math.(Crelle's Journal), to appear, math.AG/0512199.

[14] H. Konno, Cohomology rings of toric hyperkahler manifolds, Internet J. Math. 11(8), (1997), 1001-1026.

[15] N. Proudfoot, Hyperkähler analogues of Kähler quotients, PhD thesis, UC Berkeley, Spring 2004, ArXiv:math.AG/0405233. 
[16] R.P. Stanley, Combinatorics and commutative algebra, Progress in Mathematics, 41, Birkhäuser, Boston, first edition 1983, second edition 1996.

[17] A. Vistoli, Intersection theory on algebraic stacks and on their moduli spaces, Invent. Math. 97 (1989), 613-670.

Department of Mathematics

University OF BRITISH COLUMBIA

1984 Mathematics RoAD

VANCOUVER

BC, Canada V6T 1 Z2

Current Address

Department of Mathematics

UNIVERSITY OF UTAH

155 S 1400 E JWB 233

SALT LAKE CITY, UT 84112

USA

E-mail address: jiangyf@math.utah.edu

Current Address

Department of MATHEMATiCs

UNIVERSITY OF WISCONSIN-MADISON

VAN VLECK HALL

480 Lincoln DRIVE

MADISON, WI 53706-1388

USA

E-mail address: tseng@math.wisc.edu

Received OCtober 11, 2007 